\title{
Betulinic acid exhibits stronger cytotoxic activity on the normal melanocyte NHEM-neo cell line than on drug-resistant and drug-sensitive MeWo melanoma cell lines
}

\author{
PAWEL SUROWIAK ${ }^{1,2,3 *}$, MARCIN DRAG $^{4 *}$, VERENA MATERNA $^{2}$, \\ MANFRED DIETEL ${ }^{2}$ and HERMANN LAGE ${ }^{2}$ \\ ${ }^{1}$ Department of Histology and Embryology, University School of Medicine, 50-356 Wroclaw; \\ ${ }^{2}$ Lower Silesian Oncology Centre, 53-413 Wroclaw; ${ }^{3}$ Division of Medicinal Chemistry and Microbiology, \\ Faculty of Chemistry, Wroclaw University of Technology, 50-370 Wroclaw, Poland; \\ ${ }^{4}$ Charité Campus Mitte, Institute of Pathology, D-10117 Berlin, Germany
}

Received February 5, 2009; Accepted April 29, 2009

DOI: $10.3892 / \mathrm{mmr}+00000134$

\begin{abstract}
Betulinic acid is a triterpene isolated from the bark of many plants that exhibits cytotoxicity in several cancer cell lines and is capable of inducing apoptosis. In this study, we examined the cytotoxic activity and apoptotic ability of betulinic acid in the drug-sensitive (MeWo) and drug-resistant melanoma MeWo CIS (cisplatin), MeWo ETO (etoposide), MeWo VIN (vinblastin) and MeWo FOTE (fotemusine) cell lines, as well as in the normal melanocyte NHEM-neo cell line. The results show that betulinic acid exhibited significant cytotoxicity on all the cell lines. However, a sulphorhodamine B cell proliferation assay and immunocytochemical analysis of Ki67 expression revealed the strongest cytotoxicity on the normal melanocyte cell line, NHEM-neo. Flow cytometry and immunocytochemical analysis of caspase 3 expression was used to confirm cell death by apoptosis. In conclusion, betulinic acid is a potential candidate for anticancer research, and may also have an application in the cosmetics industry.
\end{abstract}

\section{Introduction}

Melanoma is considered to be the most malignant type of skin cancer. Over the last 50 years, the number of patients with this type of cancer increased 6-fold; however, the percentage of cases diagnosed during the early stages of the disease also increased significantly. In the US, the 5-year survival rate of patients diagnosed with melanoma during

Correspondence to: Dr Marcin Drag, Division of Medicinal Chemistry and Microbiology, Faculty of Chemistry, Wroclaw University of Technology, Wybrzeze Wyspianskiego 27, 50-370 Wroclaw, Poland E-mail: marcin.drag@pwr.wroc.pl

\section{*Contributed equally}

Key words: betulinic acid, melanoma, melanocyte, multidrug resistance its early stages increased from $41 \%$ in $1940-1949$ to $76 \%$ in 1973-1979. This was likely due to a better understanding and recognition of the preliminary symptoms of this type of cancer. Unfortunately, the only currently successful mode of melanoma treatment is surgery, as the benefits of adjuvant and palliative systemic chemotherapy have not been unequivocally established. Therapeutic regimens based on dacarbazine are the most commonly applied strategies for the treatment of disseminated melanoma; however, only a minority of patients obtain long-lasting response rates. Various response rates have been reported for polychemotherapy treatment protocols. Unfortunately, melanoma has a very high resistance to cytostatic drugs $(1,2)$. Data from in vitro studies suggest the presence of intrinsic biological mechanisms conferring this high drug resistance (3). High risk patients are also treated with interferon $\alpha$ as adjuvant therapy. However, this type of treatment has low levels of efficacy, high toxicity levels and high treatment cost. At present, patients with metastatic melanoma have a median survival of 6-10 months, and stage IV patients have a five-year survival rate of less than 5\% (4). Consequently, future studies on melanoma must focus on two areas of research: the establishment of a system for the early detection of melanoma, and the investigation of the resistance of melanoma cells to cytostatic drugs.

Multidrug resistance (MDR) is a complex event regulated by several possibly unrelated mechanisms, including the expression of $\mathrm{ABC}$-transporters, apoptosis dysregulation and DNA repair. In the case of metastatic melanoma, cells do not respond to applied therapy due to mutation resulting in a loss of Apaf 1 expression, which is a cofactor in the apoptosome complex (5).

Due to the complexity of MDR, current strategies aim to identify drugs active against cells resistant to classic chemotherapy. A promising group of compounds are those capable of inducing apoptosis, among them betulinic acid. Betulinic acid has attracted considerable attention due to its confirmed cytotoxicity toward cancer cells. The first study regarding betulinic acid activity against cancer cells was published in 1995, with the focus on melanoma (6). Notably, betulinic 
acid had almost no activity against keratinocytes and skin fibroblasts (7). The activity of betulinic acid against drugsensitive compared to drug-resistant melanoma cell lines has not previously been investigated in detail. Besides clarifying the activity of this compound, such a comparison allows for indirect speculation on its mechanisms of action. A lack of difference in activity against drug-sensitive and drug-insensitive cell lines would suggest a differential mechanism of action, while a difference in activity would suggest an overlapping or similar mechanism.

In this study, we compared the activity of betulinic acid in a drug-sensitive (MeWo) cell line and in several drugresistant (MeWo ETO, MeWo VIN, MeWo FOTE and MeWo CIS) melanoma cell lines, as well as in healthy melanocytes (NHEM-neo cell line).

\section{Materials and methods}

Betulinic acid. Betulinic acid was synthesized from betulin in two steps as previously described (8). The structure of the compound was confirmed using ${ }^{1} \mathrm{H}$ NMR spectroscopy and compared with previously published data (8).

Cell lines. The characteristics and culture of the human melanoma cell line MeWo and its drug-resistant variants (established by our laboratory) MeWo ETO, MeWo VIN, MeWo FOTE and MeWo CIS were previously described (Table I). The human melanocyte cell line NHEM-neo CC-2504 (Cambrex Bio Science Verviers, Germany) was used as the control.

Cell culture. Human melanoma cells were grown in Leibovitz L-15 medium (Biowhittaker, Walkersville, MD) supplemented with $10 \%$ fetal calf serum (Gibco/BRL, Grand Island, NY), $1 \mathrm{mM}$ L-glutamine, $6.25 \mathrm{mg} / \mathrm{l}$ fetuin, $80 \mathrm{IE} / 1$ insulin, $2.5 \mathrm{mg} /$ $\mathrm{ml}$ transferrin, $0.5 \mathrm{~g} / 1$ glucose, $1.1 \mathrm{~g} / 1 \mathrm{NaHCO}_{3}, 1 \%$ minimal essential vitamins and 20,000 kIE/l trasylol in a humidified atmosphere of $5 \% \mathrm{CO}_{2}$ at $37^{\circ} \mathrm{C}$. NHEM-neo melanocytes were grown using the Clonetics MGM-4 ${ }^{\mathrm{TM}}$ BulletKit CC-3249 (Cambrex) with supplements as recommended by the manufacturer.

Cell proliferation assay. Chemoresistance was tested using a sulphorhodamine B (SRB) proliferation assay. Briefly, 800 cells per well were seeded in 96-well plates in triplicate. After $24 \mathrm{~h}$ of cell attachment, betulinic acid was added in a dilution series with a 5-day incubation prior to SRB staining. Incubation was terminated by replacing the medium with $10 \%$ trichloroacetic acid followed by incubation at $4^{\circ} \mathrm{C}$ for 1 . Subsequently, the plates were washed five times with water and stained by adding $100 \mu \mathrm{l}$ of $0.4 \%$ SRB (Sigma, St. Louis, MO, USA) in $1 \%$ acetic acid for $10 \mathrm{~min}$ at room temperature. Unbound dye was eliminated by washing the plates five times with $1 \%$ acetic acid. After air-drying and re-solubilization of the protein-bound dye in $10 \mathrm{mM}$ Tris- $\mathrm{HCl}$ ( $\mathrm{pH} 8.0$ ), absorbance was read at $562 \mathrm{~nm}$ using an ELISA Reader (EL 340 Microplate Bio Kinetics Reader, Bio-Tek Instruments, Winooski, VT, USA). Measurements were carried out in triplicate in three independent experiments. $\mathrm{IC}_{50}$ values were calculated from three independent experiments for each cell line as previously described (9).
Table I. The melanoma MeWo cell line with drug-resistant sublines.

\begin{tabular}{lcc}
\hline Cell line & Selection agent & Refs. \\
\hline MeWo & - & $(22-27)$ \\
MeWo ETO & Etoposide & $(22,23,27)$ \\
MeWo VIN & Vindesine & $(22-24)$ \\
MeWo FOTE & Fotemusine & $(22,24,25)$ \\
MeWo CIS & Cisplatin & $(22-24,26)$ \\
\hline
\end{tabular}

Immunocytochemistry. The NHEM-neo cells as well as the MeWo cell line and its drug-resistant derivates were incubated for $72 \mathrm{~h}$ with $10 \mu \mathrm{M}$ of betulinic acid. Control cells of each type were grown without the compound. For the detection of caspase 3 and Ki67 proteins by immunocytochemistry, cells were seeded on slides, fixed for $10 \mathrm{~min}$ in a methanol:acetone (1:1) mixture at $-20^{\circ} \mathrm{C}$ and air-dried. Afterward, the slides were incubated for $5 \mathrm{~min}$ in $3 \% \mathrm{H}_{2} \mathrm{O}_{2}$ to block endogenous peroxidase. Immunocytochemical reactions were conducted using the following antibodies: mouse mAb AM1.31-11 (Oncogene, San Diego, CA, USA) directed against caspase 3 [dilution 1:250 in Antibody Diluent, Background Reducing (DakoCytomation, Glostrup, Denmark) for $1 \mathrm{~h}$ at room temperature] and mouse mAb MIB-1 (DakoCytomation) directed against Ki67 (dilution 1:100 in Antibody Diluent, Background Reducing for $1 \mathrm{~h}$ at room temperature). Each reaction was accompanied by a negative control (Primary Mouse Negative Control; DakoCytomation). The antigens were visualized using biotinylated antibodies (15 $\mathrm{min}$ at room temperature), the streptavidin-peroxidase complex (15 min at room temperature), LSAB+, HRP (DakoCytomation) and NovaRed (Vector Laboratories, UK) (10 $\mathrm{min}$ at room temperature). Preparations were counterstained with Mayer's hematoxylin (DakoCytomation), dehydrated and mounted.

Evaluation of reaction intensity. In the immunostained cancer cell lines, specific staining reactions were localized by two experienced histologists. Immunostaining reactions were evaluated using a simplified scale in which a score was assigned according to the intensity of the reaction ( 0 , total absence of staining; $1,<10 \%$ positive cells; $2,10-30 \%$ positive cells; $3,>30 \%$ positive cells) as described previously (10-12).

Cell cycle analysis. Cells were incubated with betulinic acid in 3.5-cm dishes. After the incubation period, cells were treated with trypsin (Biochorom AG, Berlin, Germany), washed with $\mathrm{PBS}$ at $5^{\circ} \mathrm{C}$ and centrifuged (5415C, Eppendorf, $3000 \mathrm{rpm}$ ), and the cell pellet was treated with $1 \mathrm{ml}$ of $70 \%$ ethanol (J.T. Baker, Griesheim, Germany). Prior to measurement, cells were again centrifuged and incubated with a $1-\mathrm{ml}$ solution of $0.1 \%$ Triton X-100 (Sigma, Steinheim, Germany), 0.5\% BSA (Fraction V) (Serva, Heidelberg, Germany) and $5 \mu \mathrm{g} / \mathrm{ml}$ RNase, DNase-free (Roche, Mannheim, Germany) in ultrapure water for $1 \mathrm{~h}$ at $37^{\circ} \mathrm{C}$. After centrifugation, $0.5 \mathrm{ml}$ of a solution containing $0.1 \%$ Triton X-100, $0.5 \%$ BSA (Fraction V) and $5 \mu \mathrm{g} / \mathrm{ml}$ of a fluorescent dye (propidium iodide; Sigma) in ultrapure water 


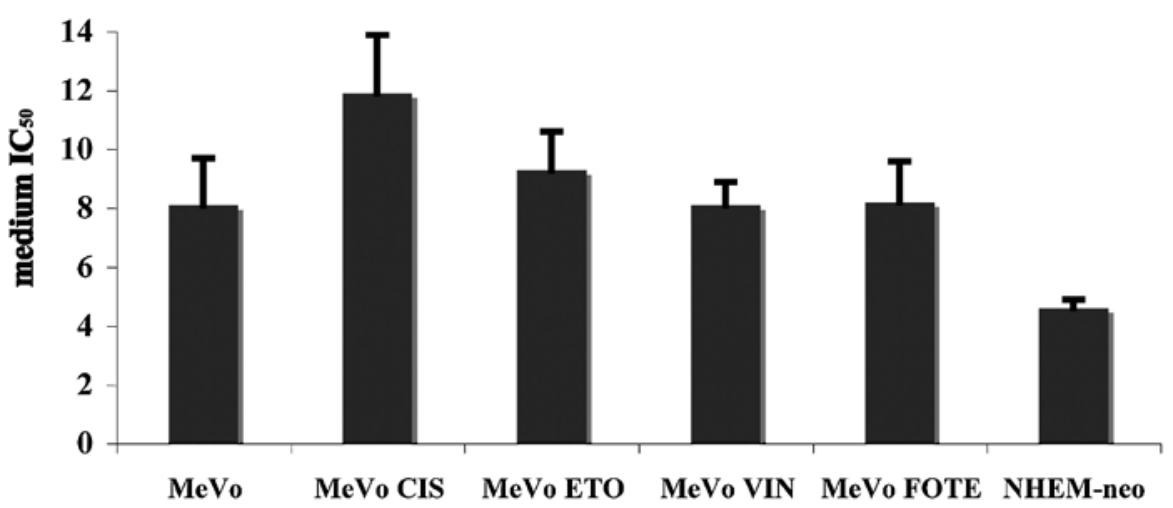

Figure 1. Cytotoxicity of betulinic acid on melanoma MeWo, MeWo ETO, MeWo VIN, MeWo FOTE and MeWo CIS cancer cell lines as well as the melanocyte NHEM-neo cell line. Cytotoxicity is represented as $\mathrm{IC}_{50}$ values $(\mu \mathrm{M})$.
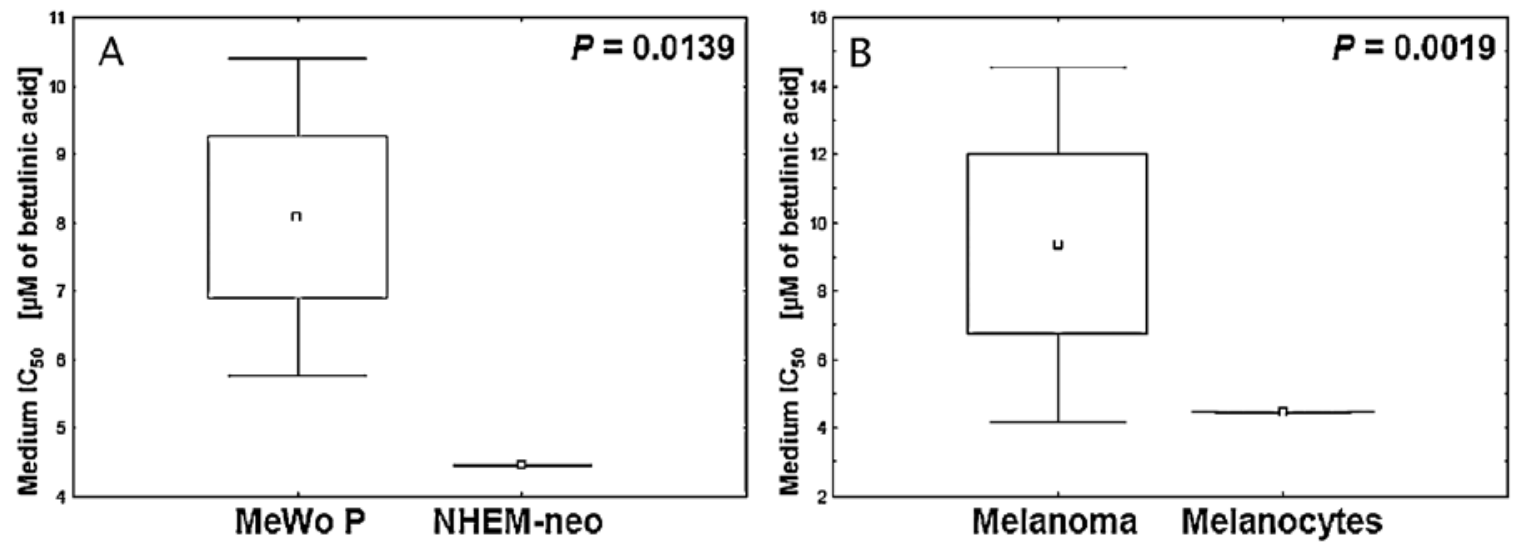

Figure 2. (A) Mean $\mathrm{IC}_{50}$ values of betulinic acid against (A) the parental melanoma MeWo cell line compared to the melanocyte NHEM-neo cell line, and (B) all the melanoma cell lines compared to the melanocyte NHEM-neo cell line (ANOVA rank test of Kruskal-Wallis).

was added to the cells. Fluorescence measurement by flow cytometry provided a measure of the amount of propidium iodide that had reacted with the cells and, indirectly, the amount of DNA content. A solution containing 5,000 cells was measured by FACScan (Becton Dickinson, Heidelberg, Germany) in FL-2 mode. Calculations were performed using CellQuest software version 1.2.2 (Becton Dickinson).

Statistical analysis. Statistical analysis was carried out using Statistica 98 PL software (Statsoft, Krakow, Poland). P-values $<0.05$ were considered to indicate a significant relationship.

\section{Results}

Influence of betulinic acid on melanoma MeWo cell lines and the melanocyte NHEM-neo cell line. We first investigated the cytotoxicity of betulinic acid on the parental MeWo cell line and its drug-resistant derivates. $\mathrm{IC}_{50}$ values for all of the tested cell lines were in the range of 8-9 $\mu \mathrm{M}$, with a slightly lower value for the MeWo CIS derivative $(11.9 \mu \mathrm{M})$ (Fig. 1). Of note, the normal melanocyte cell line NHEM-neo exhibited the strongest inhibition of proliferation as compared to the other cell lines tested $\left(\mathrm{IC}_{50} 4.6 \mu \mathrm{M}\right)$. Analysis using the ANOVA rank test of Kruskal-Wallis indicated that proliferation in NHEM-neo cells with betulinic acid was $\sim 2$-fold more strongly inhibited compared to the parental MeWo cell line (Fig. 2A) and to the mean $\mathrm{IC}_{50}$ of all of the melanoma cell lines tested (drug-sensitive and drug-resistant) (Fig. 2B).

Influence of betulinic acid on the expression of caspase 3 and the Ki67 proliferation index. In order to examine the influence of betulinic acid on the expression of the apoptotic protein caspase 3 and the Ki67 proliferation index, immunohistochemical staining was carried out in all the cell lines.

The caspase pathway is the major pathway leading cells into apoptosis, and caspase 3 is an executor of cell death that is activated when apoptosis is induced. Therefore, the activation of caspase 3 is a reliable indicator of cell death by apoptosis. In the present study, we noted a positive caspase 3 reaction localized in the cytoplasm and the nucleus (Fig. 3A1 and A2 for MeWo cells and Fig. 3B1 and B2 for NHEM-neo cells). Among the NHEM-neo, MeWo and MeWo VIN cell lines, the most significant increase in caspase 3 expression was observed in the controls, while the MeWo FOTE and MeWo ETO cell lines were in the middle range and the MeWo CIS line exhibited the weakest increase. 


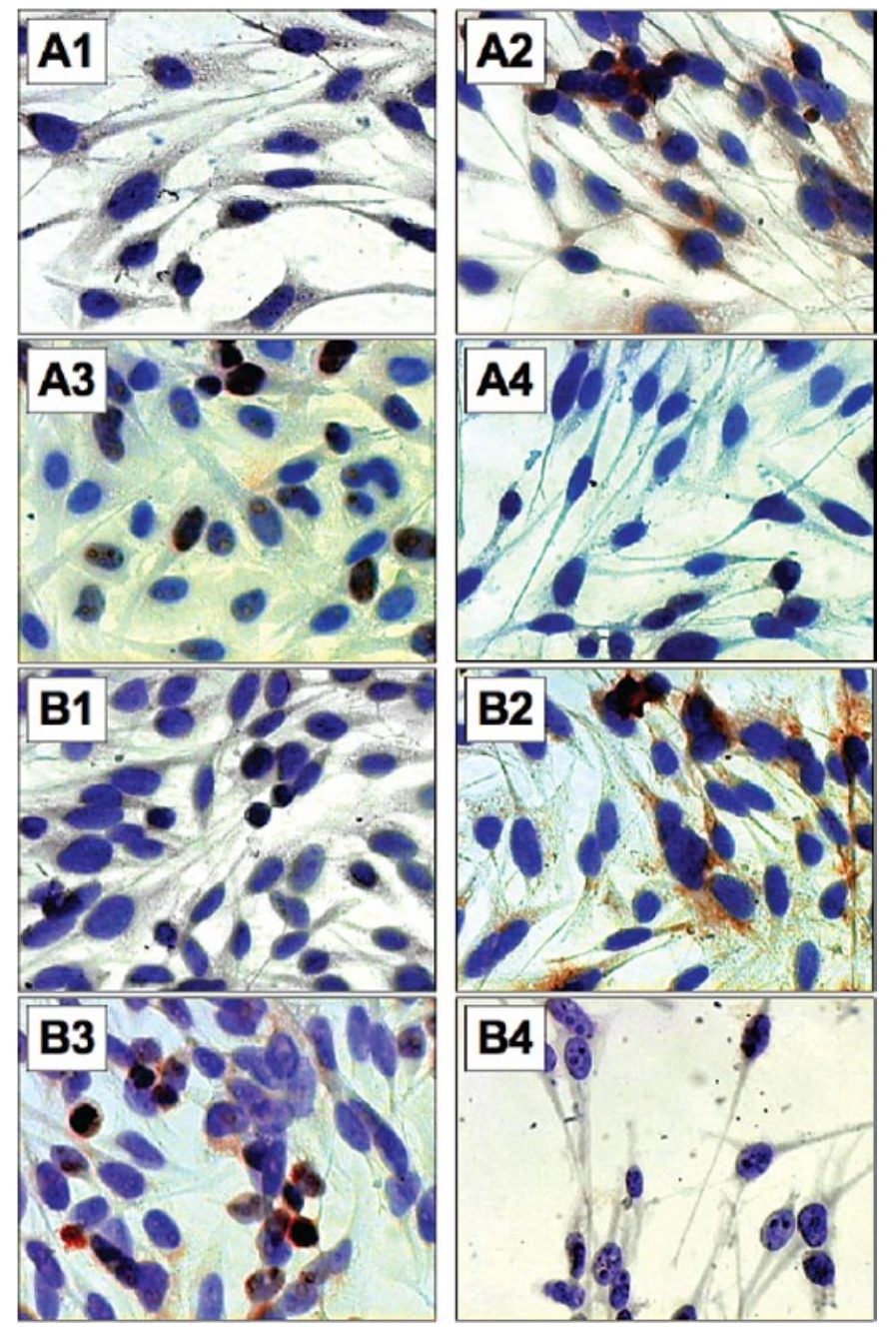

Figure 3. Immunocytochemical localization of caspase 3 expression in (A1) untreated MeWo cells and (A2) betulinic acid-treated MeWo cells. Ki67 expression in (A3) untreated MeWo cells and (A4) treated MeWo cells. Caspase 3 expression in (B1) untreated NHEM-neo cells and (B2) treated NHEM-neo cells. Ki67 expression in (B3) untreated NHEM-neo cells and (B4) treated NHEM-neo cells. Treated cells were exposed to $10 \mu \mathrm{M}$ of betulinic acid for $72 \mathrm{~h}$.

The Ki67 proliferation index is an important prognostic factor in several types of cancer. Ki67 protein is expressed in all phases of the cell cycle except the G0 phase, and serves as a good marker of proliferation. Here, the results were positive for Ki67 staining in the nucleus (Fig. 3A3 and A4 for MeWo cells and Fig. 3B3 and B4 for NHEM-neo cells). All the tested cell lines demonstrated a similar amount of decrease in Ki67 expression.

In short, $72 \mathrm{~h}$ of incubation with betulinic acid significantly decreased the expression level of Ki67 (Table II) and increased the expression level of caspase 3 (Table III).

Influence of betulinic acid on the cell cycle in the parental melanoma MeWo and melanocyte NHEM-neo cell lines. Since betulinic acid induces different biological effects in different cell types, we investigated whether it affected apoptosis by inducing cell death and cell cycle arrest in the parental MeWo cell line and the NHEM-neo cell line. Cells were treated with
Table II. Ki67 expression in the cell lines without (control) and with pre-treatment with betulinic acid $(10 \mu \mathrm{M})$.

\begin{tabular}{lcc}
\hline & \multicolumn{2}{c}{ Ki67 expression (score 0-3) } \\
\cline { 2 - 3 } Cell line & Control & Betulinic acid \\
\hline NHEM-neo & 2 & 0 \\
MeWo & 3 & 1 \\
MeWo ETO & 2 & 1 \\
MeWo VIN & 2 & 0 \\
MeWo FOTE & 3 & 1 \\
MeWo CIS & 2 & 1 \\
\hline
\end{tabular}

Table III. Caspase 3 expression in the cell lines without (control) and with pre-treatment with betulinic acid $(10 \mu \mathrm{M})$.

\begin{tabular}{lcc}
\hline & \multicolumn{2}{c}{ Caspase 3 expression (score 0-3) } \\
\cline { 2 - 3 } Cell line & Control & Betulinic acid \\
\hline NHEM-neo & 0 & 3 \\
MeWo & 0 & 3 \\
MeWo ETO & 0 & 2 \\
MeWo VIN & 0 & 3 \\
MeWo FOTE & 0 & 2 \\
MeWo CIS & 0 & 1 \\
\hline
\end{tabular}

$0,1,10$ and $20 \mu \mathrm{M}$ of betulinic acid for $24 \mathrm{~h}$. Cell cycle distribution analysis by measuring the DNA content of cells showed no influence of betulinic acid after a 24-h incubation at any tested concentration in the parental MeWo cell line (ANOVA rank test of Kruskal-Wallis, P>0.05) (Table IV), while a significant increase in the pre-G1 phase and a decrease in the $\mathrm{S}$ and $\mathrm{G} 2$ phases was noted in the NHEM-neo cells (ANOVA rank test of Kruskal-Wallis, $\mathrm{P}<0.05$ ) (Table V). The observed generation of the pre-G1 population of cells was apparently due to the DNA fragmentation of dead cells induced by betulinic acid, indicating that apoptosis might have occurred.

To confirm the apoptosis observed upon immunohistochemical staining in the parental MeWo cell line, cells were incubated for $72 \mathrm{~h}$ with $0,1,10$ and $20 \mu \mathrm{M}$ of betulinic acid. A significant increase in cells in the pre-G1 phase and a decrease in cells in the $\mathrm{S}$ phase was observed (ANOVA rank test of Kruskal-Wallis, $\mathrm{P}<0.05$ ) (Table IV). This confirmed that the cell death induced by betulinic acid was indeed due to apoptosis.

\section{Discussion}

Low-molecular-weight molecules have recently attracted considerable interest due to their activity against different types of tumors. This action is often due to the induction of cell death by apoptosis. However, the precise mechanisms of action are very often unidentified or an object of dispute. 
Table IV. Cell cycle analysis based on DNA content* in the MeWo melanoma cell lines.

$24 \mathrm{~h}$

$72 \mathrm{~h}$

\begin{tabular}{cccccccccc}
\cline { 2 - 5 } Betulinic acid & pre-G1 $(\%)$ & $\mathrm{G} 1(\%)$ & $\mathrm{S}(\%)$ & $\mathrm{G} 2(\%)$ & & pre-G1 (\%) & $\mathrm{G} 1(\%)$ & $\mathrm{S}(\%)$ & $\mathrm{G} 2(\%)$ \\
\hline $0 \mu \mathrm{M}$ & 16 & 47 & 17 & 20 & 17 & 45 & 19 & 19 \\
$1 \mu \mathrm{M}$ & 19 & 43 & 15 & 23 & 15 & 44 & 24 & 17 \\
$10 \mu \mathrm{M}$ & 15 & 46 & 14 & 25 & & 13 & 45 & 18 & 24 \\
$20 \mu \mathrm{M}$ & 12 & 49 & 16 & 23 & 58 & 22 & 13 \\
\hline
\end{tabular}

*Assessed using propidium iodide staining for flow cytometry after a 24- and 72-h incubation with $0,1,10$ and $20 \mu \mathrm{M}$ of betulinic acid.

Table V. Cell cycle analysis based on DNA content* in the melanocyte NHEM-neo cell line.

\begin{tabular}{lcccc}
\hline & \multicolumn{4}{c}{$24 \mathrm{~h}$} \\
\cline { 2 - 5 } Betulinic acid & pre-G1 (\%) & $\mathrm{G} 1(\%)$ & $\mathrm{S}(\%)$ & $\mathrm{G} 2(\%)$ \\
\hline $0 \mu \mathrm{M}$ & 17 & 40 & 19 & 24 \\
$1 \mu \mathrm{M}$ & 25 & 46 & 15 & 14 \\
$10 \mu \mathrm{M}$ & 29 & 52 & 9 & 10 \\
$20 \mu \mathrm{M}$ & 32 & 55 & 5 & 8 \\
\hline
\end{tabular}

*Assessed using propidium iodide staining for flow cytometry after a 24-h incubation with $0,1,10$ and $20 \mu \mathrm{M}$ of betulinic acid.

Among the compounds with anticancer activity are natural derivatives. These appear to be a promising tool in the treatment of various types of cancer, and several are being used in clinical trials. Betulinic acid, isolated from birch bark, is an example of a compound widely recognized as possessing these properties (13-17).

In this study, we investigated the ability of betulinic acid to inhibit cell proliferation in the parental melanoma MeWo cell line and its drug-resistant sublines MeWo ETO, MeWo VIN, MeWo FOTE and MeWo CIS, as well as in the human melanocyte NHEM-neo cell line, used as the control. In light of previous studies (7), the choice of cell lines was based on the potential effect of the administration of betulinic acid. The skin and digestion system appeared to be the most reasonable choices. The high resistance to chemotherapy of these cancer types encouraged us to investigate whether betulinic acid exhibited the same effect on drug-resistant and drug-sensitive cell lines. To date, there are few reports describing similar types of investigations. Fernandes et al (18) described the inhibition of growth and induction of apoptosis in a drug-sensitive and in a vincristine (Lucena 1) drug-resistant K562 (erythroleukemia) cell line. Jung et al (19) described the cytotoxicity of betulinic acid against both parental and drug-resistant (5-fluorouracil and oxaliplatin) colon cancer SNU-C5 cell lines. Cytotoxicity was not observed in cells resistant to irinotecan. However, the researchers in this study focused on the sensitization of the cell lines to betulinic acid, and did not provide specific data on the effect of betulinic acid on these cell lines. Additionally, Fulda and Debatin (20) and Sawada et al (21) described the sensitization of multiple cell lines to cytostatics, but did not provide any data regarding drug-resistant cell lines. Notably, our results show that betulinic acid inhibited proliferation at the same level in the parental MeWo cell line and its drug-resistant sublines (MeWo ETO, MeWo VIN, MeWo FOTE and MeWo CIS), a level similar to that reported for several cancer cell lines in previous studies. Both the SRB proliferation assay and immunocytochemical analysis of Ki67 expression confirmed similar cytotoxicity of betulinic acid on the tested melanoma cell lines. Increased expression of caspase 3 in betulinic acidtreated melanoma cells suggests that cell death occurred via the apoptotic pathway.

We also investigated whether normal cells from which melanoma originates are susceptible to betulinic acid. Notably, we found that betulinic acid most strongly inhibited proliferation in the healthy human melanocyte NHEM-neo cell line. To the best of our knowledge, this is the first study to demonstrate that cancer cell lines are less sensitive to betulinic acid than normal cell lines. In an earlier study, Zuco et al described the effect of betulinic acid on melanoma, ovarian carcinoma, non-small cell lung carcinoma, cervical carcinoma and small cell lung carcinoma (parental and resistant to doxorubicin) cell lines, as well as on normal cell lines, namely dermal fibroblasts and peripheral blood lymphoblasts. The authors observed that betulinic acid exhibited much more activity against cancer cell lines than normal cell lines (7). In the present study, flow cytometric analysis suggested that the healthy human melanocyte NHEM-neo cell line underwent apoptosis after $24 \mathrm{~h}$, while the parental melanoma MeWo cell line exhibited an increase in the pre-G1 population after $72 \mathrm{~h}$.

Our results suggest that betulinic acid can be considered a candidate for anticancer therapy. In light of our findings, the side effects of such therapy may involve an increase in normal melanocyte apoptosis, resulting in permanent or temporary albinism. However, considering that currently used cytostatic drugs with anticancer properties also have a strong effect on all proliferating cells, this observation should have a marginal effect on total therapy. Additionally, the strong activity of betulinic acid against both melanoma and melanocyte cells observed in the present study warrants further investigation for its potential application in the cosmetics industry, where it may be found to have an effect on skin hyperpigmentation or melanosis, common cosmetic defects. 


\section{Acknowledgements}

This work was supported by the Ministry of Science and Higher Education of Poland, grant 2 P05A 01330.

\section{References}

1. Ho RC: Medical management of stage IV malignant melanoma. Medical issues. Cancer 75: 735-741, 1995.

2. Barth A and Morton DL: The role of adjuvant therapy in melanoma management. Cancer 75: 726-734, 1995.

3. Schadendorf D, Worm M, Algermissen B, Kohlmus CM and Czarnetzki BM: Chemosensitivity testing of human malignant melanoma. A retrospective analysis of clinical response and in vitro drug sensitivity. Cancer 73: 103-108, 1994.

4. Balch CM, Buzaid AC, Soong SJ, et al: Final version of the American Joint Committee on Cancer staging system for cutaneous melanoma. J Clin Oncol 19: 3635-3648, 2001.

5. Soengas MS, Capodieci P, Polsky D, et al: Inactivation of the apoptosis effector Apaf-1 in malignant melanoma. Nature 409: 207-211, 2001.

6. Pisha E, Chai H, Lee IS, et al: Discovery of betulinic acid as a selective inhibitor of human melanoma that functions by induction of apoptosis. Nat Med 1: 1046-1051, 1995.

7. Zuco V, Supino R, Righetti SC, et al: Selective cytotoxicity of betulinic acid on tumor cell lines, but not on normal cells. Cancer Lett 175: 17-25, 2002.

8. Kim DSHL, Chen ZD, Nguyen VT, et al: A concise semi-synthetic approach to betulinic acid from betulin. Synth Commun 27: 1607-1612, 1997.

9. Gyorffy B, Surowiak P, Kiesslich O, et al: Gene expression profiling of 30 cancer cell lines predicts resistance towards 11 anticancer drugs at clinically achieved concentrations. Int J Cancer 118: 1699-1712, 2006.

10. Kowalski P, Surowiak P and Lage H: Reversal of different drug-resistant phenotypes by an autocatalytic multitarget multiribozyme directed against the transcripts of the $\mathrm{ABC}$ transporters MDR1/P-gp, MRP2 and BCRP. Mol Ther 11: 508-522, 2005.

11. Surowiak P, Materna V, Kaplenko I, et al: ABCC2 (MRP2, cMOAT) can be localized in the nuclear membrane of ovarian carcinomas and correlates with resistance to cisplatin and clinical outcome. Clin Cancer Res 12: 7149-7158, 2006.

12. Surowiak P, Materna V, Maciejczyk A, et al: Nuclear metallothionein expression correlates with cisplatin resistance of ovarian cancer cells and poor clinical outcome. Virchows Arch 450: 279-285, 2007.

13. Wick W, Grimmel C, Wagenknecht B, Dichgans J and Weller M: Betulinic acid-induced apoptosis in glioma cells: A sequential requirement for new protein synthesis, formation of reactive oxygen species, and caspase processing. J Pharmacol Exp Ther 289: 1306-1312, 1999.
14. Tan Y, Yu R and Pezzuto JM: Betulinic acid-induced programmed cell death in human melanoma cells involves mitogen-activated protein kinase activation. Clin Cancer Res 9: 2866-2875, 2003.

15. Hata K, Hori K and Takahashi S: Differentiation- and apoptosisinducing activities by pentacyclic triterpenes on a mouse melanoma cell line. J Nat Prod 65: 645-648, 2002.

16. Schmidt ML, Kuzmanoff KL, Ling-Indeck L and Pezzuto JM: Betulinic acid induces apoptosis in human neuroblastoma cell lines. Eur J Cancer 33: 2007-2010, 1997.

17. Fulda S, Jeremias I, Pietsch T and Debatin KM: Betulinic acid: a new chemotherapeutic agent in the treatment of neuroectodermal tumors. Klin Padiatr 211: 319-322, 1999.

18. Fernandes J, Castilho RO, Da Costa MR, et al: Pentacyclic triterpenes from Chrysobalanaceae species: cytotoxicity on multidrug resistant and sensitive leukemia cell lines. Cancer Lett 190: 165-169, 2003.

19. Jung GR, Kim KJ, Choi CH, et al: Effect of betulinic acid on anticancer drug-resistant colon cancer cells. Basic Clin Pharmacol Toxicol 101: 277-285, 2007.

20. Fulda S and Debatin KM: Sensitization for anticancer druginduced apoptosis by betulinic acid. Neoplasia 7: 162-170, 2005.

21. Sawada N, Kataoka K, Kondo K, et al: Betulinic acid augments the inhibitory effects of vincristine on growth and lung metastasis of B16F10 melanoma cells in mice. Br J Cancer 90: 1672-1678, 2004.

22. Grottke C, Mantwill K, Dietel M, Schadendorf D and Lage H: Identification of differentially expressed genes in human melanoma cells with acquired resistance to various antineoplastic drugs. Int J Cancer 88: 535-546, 2000.

23. Sinha P, Kohl S, Fischer J, et al: Identification of novel proteins associated with the development of chemoresistance in malignant melanoma using two-dimensional electrophoresis. Electrophoresis 21: 3048-3057, 2000

24. Lage H, Christmann M, Kern MA, et al: Expression of DNA repair proteins hMSH2, hMSH6, hMLH1, O6-methylguanine-DNA methyltransferase and N-methylpurine-DNA glycosylase in melanoma cells with acquired drug resistance. Int J Cancer 80: 744-750, 1999.

25. Christmann M, Pick M, Lage H, Schadendorf D and Kaina B: Acquired resistance of melanoma cells to the antineoplastic agent fotemustine is caused by reactivation of the DNA repair gene MGMT. Int J Cancer 92: 123-129, 2001.

26. Liedert B, Materna V, Schadendorf D, Thomale J and Lage H: Overexpression of cMOAT (MRP2/ABCC2) is associated with decreased formation of platinum-DNA adducts and decreased $\mathrm{G} 2$-arrest in melanoma cells resistant to cisplatin. J Invest Dermatol 121: 172-176, 2003.

27. Lage H, Helmbach H, Grottke C, Dietel M and Schadendorf D: DFNA5 (ICERE-1) contributes to acquired etoposide resistance in melanoma cells. FEBS Lett 494: 54-59, 2001. 